\title{
Kleinere Forschungen zur Geschichte des Mittelalters. XV. XVI. \\ Von
}

\author{
Paul Scheffer-Boichorst.
}

\section{Die ersten Beziehungen zwischen Habsburg und Ungarn; zurr Kritik des Banmgartenberger Formelbuches.}

Joh. Heller hat ausführlich dargethan 1), dass das Schreiben des Baumgartenberger Formelbuches Nr. 18 S. 225 aus zwei scharf zu sonderuden Theilen besteht. Der erste war auch anderweitig bekannnt, und zwar in originaler, reicherer Fassung. Rudolf von Habsburg meldete danach dem Papste Gregor, dass er nächste Ostern zum Enpfange der Kaiserkrone nach Rom aufbrechen wolle; er würde seinen Weg über Mailand nehmen. Letzteres hat der Baumgartenberger bei Seite gelassen; dafür findet sich nun in seinem Texte, als zweiter Theil, ein Bericht über eine jüngst stattgehabte Zusammenkunft Rudolfs mit dem Könige von Fraukreich: die \#in verschiedener Zeit" angeknüpften Verhandlungen, triumphirt Rudolf, hätten , endlich * ihr Ziel erreicht; zu·dem auf diplomatischem Wege getroffenen Abkommen, sich nicht gegenseitig za befeinden, sei nun bei einer persönlichen Begegnung noch hinzugefügt worden, dass sie gemeinsame Feinde haben und nur gemeinsamen Frieden schliessen wollten. Heller hat dieser Ueberlieferung vertraut. Aber wie ich in der Jenaer Literaturzeitung 1875 S. 205 ,bewies, haben weder langjährige Verhandlungen zwischen Deutschland und Frankreich stattgefunden, noch

1) Deutschland und Frankreich in ihren politischen Beziehungen vom Ende des Interregnums bis zuin Tode Rudolfs von Habsburg $147 \mathrm{ff}$.

Mittheilungen $\mathrm{X}$. 
ist an eine persönliche Begegnung des deutschen mit dem französischen König zu denken. Ich versuchte eine neue Deutung: indem ich taran ecirnerte, dass der Bandrgartenberger ins viel'ach aulere Namen überlefert hat, als die originalen Briefe, dass er z. B. awrmal deu Köuig rou Frankreich nennt, während die echten Texte rom Köunge ion Sicilien rerlen ${ }^{1}$ ), glaubte ich, auch hier sei eine solche Vertiuschung vorgenommen worden. Ich deutete len zweiten Theil des Briefe, wie er in Banugartenberger Formelbuch vorliegt, daun eben auf die sicilisch-deutsches Beziehungen. Hier trafen alle Voraussetzungeu zu; es bestand eine so genaue Uebereinstimmung der Einzelheiten. dass ich in clen Angaben des Briefes gewissermassen den natürlichen Abschluss eiuer ganz genetischen Entwicklung der deutsch-sicilischen Politik erblickte.

Zwei Franzosen. die sich in der Zwischenzeit mit unserer Frage beschäftigen mussten, haben meine Ausführungeu nicht gekannt. Dem einen, A. Leroux, ist die Zusammenkunft Rudolfs von Habsburg und Philipns von Frankreich eine feststehende Thatsache ${ }^{2}$ ); der andere, Ch. Langlois, hegt wohl Zweifel. kann sich aber doch nicht entschliessen, die Leberlieferung röllig preiszugeben ${ }^{3}$ ). Auders ein Deutscher, welcher nach unir Gelegenheit hatte, unser Schreiben zu verwerthen: A. Busson hat das Resultat meiner Forschung unbedingt angenommen und seinetwegen eine früher gehegte Meinung aufgegeben $\left.{ }^{4}\right)$. Fr werde sich wohl hütev, scherzt der Innsbrucker College, eine Ceusur, die ich etwaigem, meiner Unter'suchung sich entgegenstellendem Zweifel angedroht hätte, auf sein Haupt zu laden. Und doch - -

Meine Schlussfolgerung lässt Ein Moment ausser Acht: ganz geblendet, dass Alles so vortrefflich in einander greift, wenn man unseren Brief auf Rudolf von Habsburg und Karl von Sicilien deutet, habe ich gar nicht erwogeu, ob nicht die Beziehungen Rudolfs zu einem andereu König eine analoge Entwickelung genommen haben. Der Gedanke möchte wohl den Wenigsten gerade sehr vahe liegen, und die Wahrscheinlichlseit hat er von vorveherein wicht für sich. Indess wird sich zeigen, dass die Wirklichkeit zu seines Gunsten entscheidet.

Eins wurde bisher immer übersehen: der zweite Theil unseres Schreibens, um den sich eben die Untersuchung dreht, war längst als selbständiges Stück gedruckt, nämlich bei Gerbert Cod. epist. 149

1) Heller 152 Anm. 1. $\quad$ 2) Recherches critiques sur les relations politi-

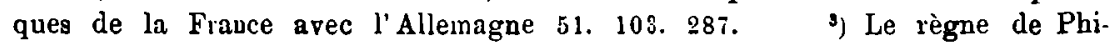
lippe III. le hardi $84 . \quad$ † Die Ides des deutschen Erbreichs. Wiener S. B. LXXXVIII. 680 Anm. உ vgl. 4. 
Nr. 7 und Cenui Mon. pont. II. 410 Nr. 31, nicht aber als ein Brief, der über Karl von Sicilien, natürlich ebensowenig ïber Philipp von Fraukreich handelte, der vielmelr: eine Zusammenkunft Rudolfs mit Ladislaus IV. von Ungarn betrifft. Die Ueberschrift lautet: „Rudolfus cuidam siguificat conditiones foederis inter ipsum et Ungariae regen initas, " und wo der Baumgartenberger schrieb: „ cum magnifico principe, rege videlicet Francie, habe Rudolf sich verbündet, - heisst es in dem anderen Texte: „cum domivo I., " worunter eben nur Ladislaus IV. von Ungarn verstanden sein kanu. Doch es finden sich noch audere Verschiedenbeiten. Geradeso wie der Baumgartenberger den ersten Theil des Briefes verstümmelt hat, wie er hier Rudolfs Reise über Mailand bei Seite liess, so scheint er dich auch im zweiten gekürzt zu haben. Nach ihm hätten Rudolf und seiu Freund bei ihrer Zusammenkunft dem früheren Bunde die neue Bestimmung hinzugefügt: , quod in litibus et questionibus, in bellis publicis seu privatis atque occasione qualibet, (in quae) et nunc vel in autea impellemur, unus alterum tam fideliter quam viriliter adiuvabit uec alter sine alterius beneplacito et consensu cum huiusmodi turbatore treugas, pacem vel concordiam celebrabit;" in der anderen Fassung lautet der Satz: ,quod in litibus et questionibus, super damuis datis et iniuriis irrogatis vel aliis quibuscunque, quas habemus adversus illustrem"regem Bohemiae, unus alterum tam fideliter quam viriliter adiuvabit nec unus sine alterius beneplacito et consensu cun praedicto rege treugas, pacem vel concordiam celebrabit." Dort eine ganz allyemeine Bestimmung, hier eiu Bund gegen Böhmen. Daun schliesst der Brief in Baumgartenberger Formelbuche mit der Bitte, dem Ueberbringer ein geneigtes Ohr zu leihen; bei Gerbert und Ceuni steht am Ende der abgebrochene Satz: "Caeterum de terminis terrarum nostrarum legaliter distinguendis et distinctis, in pace et concordia observandis, taliter duximus ordivandum ctc." Soweit die Verschiedenheiten beider Texte. Welchem sollen wir uus auschliessen?

Doch bevor ich in die Untersuchung eintrete, muss ich zunächst bemerken, dass die durchgehende Uebereinstimmung des doppelten Textes, die dem Herausgeber des Baumgartenberger Formelbuches Bärwald, dann Heller, mir, Busson, Leroux und Langlois entgangen war, von einern meiner Zuhörer beachtet wurde, von Herrn P. Broichmanu, dem also auch das Verdieust gebührt, die Forschung auf eine andere und - wie ich denke - auf die richtige Bahn geleitet zu haben.

Es ist die Frage, ob Rudolf anch mit Ungarn, wie mit Anjou, „zu verschiedenen Zeiten " verhandelt hat, ob ,eudlich " ein Bündniss 
zu Stande kam, ob dieses in persönlicher Begegunng beider Herrscher jene Bekräftigung und Erweiterung erfahren konnte. izucolí unci Ladisluus hatten in Cttukar ros Böduen ihrea gemeinsamen Feind. Rudolfs Beziehungen zu demselben sind uns Deutschen bekanut; hinsichtlich des Ungarn bemerke ich, dass er die Feindschaft seines Vaters gegen Ottokar geerbt hatte, dass er ihn noch im Mai 1274 seinen Erzfeind nennt ${ }^{1}$. Da wäe es doch merkwürdig gewesen, wenn er lange gezögert hätte, mit Rudolf Fühlung zu gewinnen. In der That, schon sebr bald hat er für seineu kaun achtjährigen Bruder Andreas im Hause Habsljurg eine Braut gesucht: er jauchat Rudolf entgegen, wie eimem neu aufgehenden Gestirn; er weiss noch nicht einmal, ob Rudolf überhaupt eine verfügbare Tochter besi(zt $\left.{ }^{2}\right)$; er ist desshalb auch zufrieden, wenn eine Anverwandte Rudolfs seinem Bruder versprochen wird ${ }^{3}$ ); daraus geht doch herror, dass Rudolf erst eben zum Throne gelangt ist. Zugleich beauftragt Ladislaus den Grafen Meinbard ron Tyrol, in der Verlobungs-Angelegeuheit als Sachwalter zu bandeln ${ }^{4}$ ). In beiden Briefen nimmt er die Sendung eines besonderen Boten in Aussicht, wio es doch scheint, eines Laien ${ }^{5}$. Ob derselbe in der That entsandt worden ist? Nichts steht der Annahme entgegen, und an sich ist wahrscheinlich, duss wicht gleich die erste Verhaudlung zun Ziele führte ${ }^{\mathrm{b}}$ ). Jedeufalls

1) Regesta Bobemiae II. $\delta € 1$ N. $884 . \quad$ 2) - inter filiam vestram principaliter si extat, aut filii vestri etc. 3) Palacky Ueber Formelbücher $819 \mathrm{~N}, 112$. Weniger gut bei Gerbert Cod. ep. Rud. 150 Anm. Bodmann Cod. ep. Rudolf 49 N. 47. Baumgarteuberger Formelbuch $805 \mathrm{Nr}$. 8. Andere haben diesen und die näcbslfolgenden Briefe in eine spätere Zeit gesctzt; doch ohne ausreichenden Grund. Vgl, auch Huber Gesch. Oesterreichs I. 59; Anm. 1. ) Gerbert l. c. 149 Anm. Bodmann 1. c. 47 N. 46. Baumgartenberger Formelbuch 508 N. 7. Auch hier: ,eiugdem Rom. regis, si extat, flia." Nebenbei bemerkt, kann aus den inneren Streitigkeiten Ungarns, von denen die Rede ist, für die Abfassungszeit Nichts geschlossen werden, denn 127s, 1274 und wieder $127 \mathrm{j}$ kamen Empörungen zum dusbruch. Huber im Archiv für öst. Gesch. LXV. 19t-197. 5) - nobilem rirum, magistrum N. familiarem et fidelem nostrum. Der Titel, Magister entacheidet nicht für den geistlichen Stand; ,nobilis" und, fidelis" sind Epitbeta der Laien. Zu ,Ma. gister* kann man nach ungarischem Spracbgebrauch, dapiferorum* oder, tavernicorum " hinzudenken. In letzterem Falle wäre Joachim Pectari selbst, von dem wir hören werden, dass er eifrigst die Verlobung belrieb, der Bote gewesen. 8) Dazu stimmt auch, dass in einem Glückwunschschreiben bei Bodmann l. c. 29 N. 24 von der Verlobung geredet wird, als von, sperata diutius unione." Dann hat der Papst, welcher damals allerdings in Lyou weilte, dem Plaue zugestimmt. So nach dem S. 85 Aum. 2 auzulührenden Schreiben, aber auch nuch unserer Gratulation. Wenn es hier heisst, dass alle Gegensätze , adversus Romani culminis unum caput se elevant et extollunt, " so sieht man wohl, dass der deutsch. 
war der ungarische Bote, vor welchem die Verlobung zn Stande kam, ein Geistlicher, der Scholaster von Fünfkirchen 1). Ladislans, nun über Rudolfs Familienverhältuisse unterrichtet, hatte um eine Tochter oder Nichte gebeten. Rudolf aber willfahrte gern, er gab zur Antwort ${ }^{2}$ ), dass er nicht aus seiner weiteren Verwandtschaft ausgewählt, sondern dem Bruder des Königs die eigene Tochter bestimnt habe, die Clementia; der Papst habe dem Plane zugestimmt, und die Verlobung sei schon vollzogen ${ }^{3}$ ); er schicke auch seinerseits Boten, denen der König ein gnädiges $\mathrm{Ohr}$ leiheu möge, in his quae negotium ip um contingere dinoscuntur et quae alia quaevis auxilii mutui et confoederationis alternae solatia spectant *4). Die letzteren Worte enthalten meines Wissens die erste Direktive für das spätere Bündniss. Nın aher erfolgte ein jäher Werhsel. Denn der eigentliche Urheber des Planes, der Oberschatzmeister Joachim Pectari, erhob sich gegen Ladi.laus; im Herbst 1274 wurde er gestürzt ${ }^{5}$ ), und die ungarische Politik, die Pectari offenbar in deutschfreundlichem Sinne geleitet batte, schlug die entgegengesetzte Richtung ein: sie näherte sich Böhmeu; ein vorläufiges Abkommen wurde getroffen; Karl ron Anjou sollte für Ungarn, Heinrich von Baiern für Böhmen vermitteln; auf einem Kongresse, Michaeli 1275, sollte der definitive Friede geschlossen werden; schon jetzt aber musste sich der Ungar verpflichten, ohne Böhmens Genehmigung keine Verwandtschaft mit Habsburg einzugehen, $d$.h.also den besprochenen Eheplan fallen zu lassen ${ }^{6}$ ). Indess kam der Kongress nicht zu Stande, denn Joachim Pectari, im Sommer 1275 wieder zu Gnaden aufgenommen ${ }^{7}$ ), wusste ihn zu vereiteln ! überhaupt ist Joachim jetzt erst recht die Seele der ungarischen Politik, die natïrlich in die alten, dem Böhmen feindlichen Bahnen zurücklenkt ${ }^{8}$ ). Doch hat es auch in jener Zeit, da Joachim als Rebell galt, uicht au allen Verbandlungen zwischen Habsburg und Ungarn ge-

böhmische Krieg in Sicht, aber noch nicht ausgebrochen war. Der Schreiber meint, die Verlobung würde jede Bewegung im Keime ersticken.

1) Palacky l. c. $\$ 19$ N. 118. Leider unvollständig. \%) Bodmann 1. c. 69 N. 66. Dass dieser Brief die Antwort auf den vorhin angeführten ist, kann nicht 2 weifelhaft sein. Rudolf nennt den ungarischen Boten, honorabilem virum, gibt ihm also die Titulatur eines niederen Geistlichen. of - sponsalia iam contracta spopondimus etc. - Vgl. S. 87 Anm. $\delta$. † Von Ladislaus sagt Rudolf: - peroptatis ut - inter nos perpetui foederis unio et immarescibilis amicitiae pal. mites adolescant." 8) Vgl. Huber im Archiv für öst. Gesch. LXV. 196. ๑) Voigt Urkundl. Formelbuch des Hen. Ital. 53 . Ueber die Zeit vergleiche Huber Oest. Gesch. I. 596 Anm. 2. 7) Huber im Archir a. a. O. 198. 9, S. Ottokars Brief bei Dolliner Cod. ep. Primislai 55 . 
fehlt: am 10. Mai 1275 beantwortete Gregor X. ein undatirtes Schreiben Rudolfs'), der ihm gemeldet hatte "super nuntiorum Hungariae et Bnhemiae regum leçationib'ss nobie pronositin et nblation:bus editis". Nur wird es sich hier um gemeinsame Vorschläge der damals verbündeten Könige gehandelt haben. Jetzt, als vielbedeutender Minister, hatte Joachim die freundlichen Beziehungen zu Böhmeı gelöst; und da wird sich Ungarn wieder mit Habsburg zusammengefunden haben. Freilich noch einnal schwankte das Verhältuiss; die näheren Umstände entzieheu sich unserer Kenntniss; genug, wir haben Briefe rom 22. April und 24. September 1276, wonach Ort und Zheit angesagt sind, dass der Friede zwischen Ungaru und Böhmen, der nach Ottokars Wuusch sich doch sicher gegen Habsburg richteu sollte, neuerdings bekräftigt werde ${ }^{2}$ ). Aber auch diese Kongresse haben schwerlich stattgefunden, der erste nicht, weil dann der zweite doch überflüssig gewesen wäre, der zweite nicht, weil er offenbar durch die Ereignisse überholt wurde. Rudolf hatte seinen ersten Krieg gegen Böhmen hegonnen, - und Ladislaus und Ottokar sind von einer Verständigung weit entfernt. Unter den Grüncleu, die den Böhmenkönig bestimmt haben sollen, den Kampf gegen Rudolf aufugeben, wurde auch geltend gemacht, Ladislaus hätte dem Letzteren ein starkes Heer zuführen wollen ${ }^{3}$ ). Rudolf selbst aber bestinimte, als er im November mit Böhmeu Frieden machte, dass Lngarn in demselben eingeschlossen sein solle, bis völlige Freundschaft zwischen Ladislaus und Ottokar hergestellt wäre, dass Böhmen indess alle entrissenen Burgen an Ungarn zu erstatten habe ${ }^{4}$ ). Wie man sieht, waren die Beziehungen Ungarns za Böhmen ebenso feindlich, als sie zu Habsburg freundlich waren. So blieb es auch ferner. Wir wissen, dass Ottokar sich nicht an jede Bestimmung des Friedens band, auch nicht an diejenige, welche über die Burgen handelte; es kam zu neuen Reibereien, und als dieselben am 6. Mai 1277 beigelegt wurden ${ }^{5}$ ), liess Rudolf die früheren Bestimmungen wiederholen. Danach muss doch \%wischen ihm und Ladislaus vielfach verhandelt worden sein. Ja, man wird nicht zweifeln dürfen, dass noch vor Ausbruch oder bei Beginn des deutschböhmischen Krieges eine Vereinbarung getroffen sei, wenn auch nur mit nächster Beziehung auf die Besitzungen, die Ladislaus von Ottokar

1) Gerbert 68 N. \&. Bodmann 184 N. 8. Baumgartenberger Formelbuch 879. Die chronologische Bestimmung S. 874 Anm. 1. 2) Fejér Cod. dipl. Hungariae V b 312. \$23. Den ersten Brief setzt Fejér, allerdings mit, 1276 ${ }^{2}$, zu den Dokumenten des Jahres 127:. Man vergleiche deshalb Archivio stor. ital. a a serie, XXV. 32. $405 . \quad$ s) Cont. Vindob. M. G. SS. IX. 708. औ) M. G. LL. II. $408 . \quad$ 5) M. G. LL. II. 414. 416. 
rerlangte; und dazu stimmt, dass Rudolf im Juli 1277 dem Ungarn rerspricht, er wolle ihm balten, universa et singula pacta", die er früher nit Rüchsicht auf Böhmen ühernommen habe. Der Ausdruck scheint den Friedensschlüssen vom November 1276 und Mai 1277, welche einfach dem Ungaru die ibm von Ottokar entrissenen Burgen sichern, loch nur wenig zu entsprechen, sondern auf besondere Abmachungen zu cielen. Dabei kann es denn auch an Friedens- und Freundschaftsbetheuerungen nicht gefehl $t$ haben, und in diesem Zusammenhange rerweise ich auf ein Mandat, das Ladislaus Fnde des Mai 1277 zu (iunsten der deutschen Kaufleute ergehen lässt. ,cum pacem, unionem et concordiam, quae inter nos et domiuum regem Romanorum, amicum nostrum, extitit ordinata seu statuti, perpetuo et inviolabiliter velimus observare ${ }^{1}$ ). Eine ähnliche Vergiinstigung hat auch Kudolf, in unbekaunter Zeit, den ungarischeu Kaufleuten ertheilt. Aber, der Friede, die Einigung und Eintracht", welche danach hergestellt und festgesetzt sein soll, war wohl uur eine vorläufige oder bezog sich nur auf gewisse Punkte, wie also auf die von Ottokar besetzten Burgen Ungarus. Deun erst Anfangs Juli 1277 brachten Boten, die Ladislaus nach Wien geschickt hatte, einen endgiltigen allgemeineren Bund zum Abschluss ${ }^{2}$ ). Die ungarischen Diplomaten schwuren, dass ihr König den zu entsendenden Boten Rudolfs eine gleichlautende Urkunde aussteilen würde, wie die, welche sie jetzt in die Heinat mitnahmen. Besonders wurdeu über die Vermählung des immer noch sehr jugendlichen Prinzen mit Rudolis Tochter neue Abmachungen getroffen ${ }^{3}$ ). Genug, es ist lange Zeit hindurch zwischen Habsburg und Ungarn verhandelt worden, und die Worte unseres Briefes: „die Vereinbarungen seien, hinc inde per nostros consiliarios diversis temporibus inchoatae et tandem utriusque nostrum patentibus literis approbatae" können doch auch recht gut auf die deutsch-ungarischen lleziehungen gedeutet werden, uur haben wir über die deutsch-sicilischen, denen sie freilich nicht minder gut entsprechen, eine viel genauere Kunde.

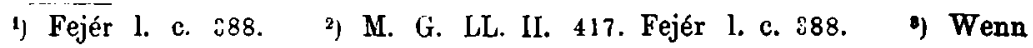
O. Lorenz Deutsche Gesch. II. 160 sagt, die Verlobung babe, , wie urkundlich sichergestellt ist, erst am 12. Juli 1277 stattgefunden, " so muss ich doch widersprechen. In dem Vertrage heisst es: sponsalıa contracta - matrimunio subsecuto die ad hoc per Ladislauum - et per nos rel per intermedios - statuendo laudabiliter consummentur. Da ist also keineswegs gesagt, dass die Verlobung erst jetzt gefeiert worden sei. Das war längst gescbehen. und zwar vor einem einzigen Boten, dem Scholaster von Füutkirchen, welcher an der aus Mehreren bestebenden Gesandtscbaft, die jetzt den Vertrag zu Stande brachte, gar keinen Antheil batte. Vgl. S. 85 Anm. ¿. 
Es ist dann die Frage, ob auf einer persönlichen Begegnung Rudolfs und Ladislaus' der Bund in angegebener Weise erweitert werden konnte.

Anfangs November 1277 kamen beide Herrscher in Haimburg zusammen ${ }^{1}$, und auf dieses Ereigniss ist denn auch der Brief, wie er bei Gerbert und Cenni vorliegt, immer bezogen worden. Man batte sich im Juli 1277 gelobt: "ut utrinque aurilio, consilio et farore omnibus et per omnia nobis invicem assistamus, cum id tempus, locue vel opportunitas vel necessitas vel utilitas nostri vel nostrorum exegerit s. Es ist natürlich etwas Anderes, es geht über den angeführten Artikel hinaus, weun auf der persönlichen Zusammenkunft, wie es bei Cenni und Gerbert heisst, ein gemeinsamer Krieg gegen Böhmen in bestimmteste Aussicht genommen wurde. Erst recht wird der Bund erweitert durch die Verpflichtung, dass der Eine obne den Auderen keinen Frieden schliessen wolle. So würde denn Gerbert's und Cenni's Text den deutsch-ungarischen Verhältnissen entsprechen.

Noch bleibt die Vereinbarung über die streitigen Grenzen, die also auch ein Zusatz zu dem vorausgegangenen Vertrag sein soll. Leider wissen wir nicht, was bestimmt worden ist, denn der Text bricht mitten im Satze ab. Jedenfalls bestanden aber Grenzstreitigkeiten, und diese hatten im Juli-Vertrage auch Berücksichtigung gefunden. Es war da festgesetzt worden, dass die alten Grenzeu erneuert werden sollten "certis ad hoc utrinque baronibus et fidelibus deputatis. Zur Zeit der Begegnung war nun zum Theile schon entschieden, sollte zum anderen Theile noch entschieden werden ${ }^{2}$ ); und das neue Debereinkommen möchte bezweckt haben, die schon vollzogene oder noch bevorstehende Grentregulirung zu sichern, würde also durchaus eine Ergänzung des früheren Vertrages gewesen sein.

Die beiden Zusätze, wie sie nach Gerbert und Cenvi auf der persönlichen Begeguung getroffen wären, stimmen nun aber gar nicht zu den Beziehungen zwischen Rudolf von Habsburg und Karl vou Aujou. Beide sind zur Zeit der böhmischen Kriege von einer Verständigung noch weit entfernt, und dann haben wohl manche Differenzen die Herrscher von Deutschland und Sicilien getrennt, aber soweit ich sehe, doch keine Grenzstreitigkeiten ${ }^{3}$ ).

1) Cont. Vindob. 1 c. 709.

2) Rudolf schreibt: de terminis terrarum nostrarum legaliter distinguendis et distinctis.

s) Noch beinerke ich, dass zwischen dem Briefe, wie er bei Gerbert und Cenni vorliegt, und dem Ver. trage, den Rudolf im Juli 127 i mit Ladislaus eingieng, eine wörtliche Ueber. einstimmung besteht. Hiernach wollte Rudolf den Böhmen ermahnen, dem Un. garn genug zu thun, de dampnis datis, iniuriis irrogatis"; auf der 
Ich meine auch jetzt noch, dass Alles vortrefflich in einander griff, so lange man an Rudolf von Habsburg und Karl von Anjou denken durfte. Das gestattete die Fassung des Baumgartenbergers, lässt sich aber mit Gerberts und Cennis Text nicht vereinen. Und da ist nun zu beachten, dass sich der Schreiber des Codex, dem Cenni und Gerbert folgen, niemals solche Aenderungen erlaubt, wie der Baumgartenberger. Dieser schweisste die zwei Briefe zu einem zusa!nmen, jener hält sie getrenut; dieser hat im ersten den Plan Rudolfs, zunächst nach Mailaud zu reisen, bei Seite gelassen, jener ihn beibehalten; und wenn hier im zoreiten uicht ron Ladislaus voi Ungarn, als Bundesgenossen, dann uicht von Böhmen, als gemeinsamem Feinde, die Rede ist, wohl aber dort, so wissen wir jetzt, wie das Urtheil zu lauten hat. Noch ein Wort über den König ron Frankreich, der mit Rudolf nach dem Baumgartenberger zusanmengekommen sein soll! Zweimal hat der Schreiber ihn statt des Königs von Sicilien genannt, hier hat er ihn an Stelle des Ungarn gesetzt: desselben Quiproquo hat er sich aber auch noch ein anderes Mal schuldig gemacht S. 354 Nr. 5

\section{Zar Geschichtschreibung von Cremona.}

Die historischen Aufzeichnungen, woran es während des 12. und 13. Jahrhunderts in Cremona keineswegs gefehlt kat, erfuhren eiu recht ungünstiges Geschick. Ich will nicht davon reden, dass ein Annalenwerk förmlich mitten entzweigerissen wurde, dass nur die erste Hälfte in die Monumenta Germaniae gelingte ${ }^{1}$ ), während die. zweite Hälfte längst an anderer Stelle gedruckt war ${ }^{2}$ ), dort aber sozusagen ein fast unbeachtetes Leben führte; - Anderes ist viel mehr zu bedauern. Dahin gehört schon, dass der zweite 'Theil der erwähuten Annalen keineswegs vollstäudig auf uns gekommen ist ${ }^{3}$ ); dahin gehört dann vor Allem der Verlust der zeitgenössischen Geschichte des Johann von Cremona. Gewiss sind es nur Bruchstücke, die der Chronist von Ursperg gerettet hat; das ganze Werk Johanns würde uns noch mauche Aufschlüsse gewähren, einerseit.s über den Verlauf

\footnotetext{
Begegnung haben nun Rudolf und Ladislaus zu dem früheren Bunde hinzinge fügt, dass sie sich gegen Böhmen beistehen wollen, superdamnis dat is et iniuriis irrogatis." Man erkennt auch darin die innige Zusanmengehörigkeit der beiden Actenstücke.

1) M. G. SS. XVIII. $800-807$ bis zum Jabre 1282 .

2) Voin Jahre 1282 bis 1269 Arch. stor. ital. Nuova serio IIIb $22-28$. 8) S. 28 , Reliqua desi. derantur." Sonst findet sich nur noch S. 16 Anm. 2 eine dürftige Notiz über die Quelle der Publikation.
} 
des grossen Schismas, das in der zweiten Hälfte des Jahrhunderts die Welt spaltete, anderseits über den Lombardenbund. Das ist wohl der grösste Verlust, den die Geschichtschreibung von Cremona erliute, iat, heiueswegs aber der eiuzige, den wır neben der Verstümmelung des augeführten Aunalenwerkes beklagen müssen. Ich möchte hier auf zwei andere Quellen Cremoneser Ursprungs verweisen, die heute rersiecht oder doch nur in Ableitungen auf uns gekommen sind.

Bisher pflegte man den recht schützenswerthen Nachrichten, die wir zu Anfang der Annal. Placent. Guelfi lesen, insgesammt eineu originalen Werth zuzuschreiben. Doch mit Unrecht; ein Theil ist entlebnt. Den Charakter als Excerpt verräth schon 1088, sofern hier auf entsprechende Angaben für spätere Zeit vorbereitet wird, ohne dass die Erwartung erfüllt würde. „1088 Prima guerra Creme fuit, " aber die folgenden Kriege fehlen. Und da werden wir denn sogleich auf die schon erwähnten Annalen ron Cremona geführt. Sie setzen den ersten Kricg gegen Crema zu 1098, sie fahren dann fort: „1130 Secuuda guerra de Crema. 1157 Tertia guerra de Crema." Und mit denselbeu Annalen lässt sich nun, falls nicht recht eigentlich Piacentiner Augelegenheiten zur Sprache kommen, durchgehende Uebereinstimmung darthun. Z. B.:

Annal. Placent.

1107 in vigilia 8. Bartholomei Cre. mona cum Papia et Laude incenderunt burgum Terdone.

1111 in vigilia s. Imerii fuit discon. fita de Brixanore. Eodem anno ultima ebdomada Madii Laudenses capti fuerunt.

1113 focus s. Iaurentii fuit.

1120 Prima guerra Parme, prelium ibi in glarea') fuit de mense Junii.

1126 Civitas Cumarum capta fuit, et codem anno mense Deceubri Padus ge. avit.

Die letzten Uebereinstimmungen meine ich 1158 und 1159 zu erkennen. 1158 entspricht dem Berichte des Cremonesers: , Mediolanum obsedit et ibi steterunt per quinque septimanas - fecit concordiam "

1) , in Garea M. G. 1. c. 412 . Vgl. die folgende Anm. 2), in glaro* 1. c. 800 . Wie zu lesen sei, ersieht man aus der Chronik des Bischofs Sicard yon Cremona: „in Parmensi glarea.* Muratori SS. VII. 595. 
der des Piacentiners „Mediolanum - obsedit et stetit in eadem obsidione per quinque septimanas - fecit concordiam. 1159 weichen beide Ueherlieferungen allerdings darin von einander ab, dass es in der Cremoneser richtig heisst, Crema sei -per septem menses" belagert worden, dass nach der Piacentiner Kaiser Friedrich es ,per uovem menses" tingeschlossen hielt. Aber in den Zahlen, namentlich deven der Jahre'), findet sich mauche Verschiedenheit, uud daun scheint mir für den auch 1159 noch bestehenden Zusammeuhang die Tagesangabe zu sprechen: nach beiden Anualisten fiel ('rema "in conversione s. Pauli “, wïlırend es sich thatsächlich nicht schon am $2 \%$, sondern erst am 26. Januar ergab ${ }^{2}$ ).

In welchem Verhältnisse nun unsere Annalen z" einauder stehen, kann nicht zweifelhaft sein. Schon der Umstand, dass die Uebereinstimmung nicht weit über die Anfünge hinausgeht, scheint die Annahme, der eine Autor habe das Werk des audern benutat, nicht eben zu empfehlen. Dann können die Annalen von Piacenza nicht Quelle sein, weil sie zu ihrem Satze "Prima guerra Creme fuit," wie gesagt, das in Aussicht gestellte Correlat nicht bieten, während in den Cremoneser Aunalen die erwarteten Kriege folgen: ,1130 secunda guerra de Crema. 1157 tertia guerra de Crema. "Wollte dagegen Jemand die Aunalen vou Cremona als Quelle bezeichnen, so liesse sich auf die oben schon angeführte Notiz zu 1113 hinweisen: „focus s. Laurentii fuit." Diese Kürze macht die Angabe eigentlich nur für einen Cremoneser verständlich, und zwar für einen Cremoneser, der sich des grossen, für Cremona verhänguissvollen Brandes vom Tage des hl. Laurenz noch zu entsinnen wusste. Wenn der Piacentiner das Sätzchen richtig verstanden hätte, würde er es wohl gauz bei Seite gelassen haben. Jener Cremoneser aber, der mehr als ein Jahrhundert nach 1113 schrieb, meinte seinen Leseru eine Erklärung schuldig zu sein. Aus seiner, jeden Zweifel hebenden Umschreibung: "Cremona fuit incensa 1113 in festo s. Iaurentii " hätte gewiss nie ein Piacentiner gemacht: ,focus s. Laureutii fuit. “

Damit ist denn zugleich auch gesagt, dass die deu beiden Annalen zu Gruude liegenden Notizen in Cremona entstanden sind. Die mehrfache Hervorhebung Cremonas, die ich nicht weiter verfolgen will, führt zu dem gleichen Ergebniss. Und so sind deun kurze, aber in

ग) Annal. Cremon. $1098=$ Annal. Placent. 1088. Annal. Cremon. $1016=-$ Annal. Placent. 1026, und Anderes. Die Beschaffenbeit der gemeinsamen Quelle, auf die wir geführt werden, muss zu solchen Irrthümern, wie sie hier auf einer Seite vorliegen, leicht veranlasst haben. gewin IV. is ed. Waitz p. 258. 
den Tages- oder Monatsangaben recht genauc Aufzeichnungen, die wahrscheinlich bis 1159 reichten, fiir dic Historiographic von Cremona in Anspruch zu nehmen.

Der Cremuneser Úrsprungt orinnert uus an den bekannten Chronisten von Cremona, au Bischof Sicard. Uud auch er nun hat aus der verlorenen Quelle geschöpft, doch gemäss der Natur seine. knappeu Compendiums der Weltgeschichte, nicht eben oft. Die uns erhaltenen Annaleu ron Cremona könuen seinen Angabeu nicht zu Grunde liegen, denn sic entstanden erst nach seiuem Tode D.ısselbe gilt ron den Piacentiner Aunalen. A ber auch unsere Cremoneser und Piicentiner Anualisten huben sich uicht seines Werkes bedieut, schon deshalb nicht, weil sie genauer unter sich ïbereiustimmen, als mit ihm.

Sicard. 58f, 594. 505. Placent. Crenon.

1107 Cremonenses, Lau. $110 i$ in vigilia s. Cremonenses Laulenseset denses, Papienses incende jBarth. Cremona cum $\mathrm{Pa}$ |Papienses incenderunt burrunt burgum 'Terdonae in pia et Laude incenderunt'ghum Dertone in $\mathrm{vig}$ ilia mense Auguato.

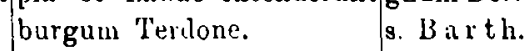

1110 Eodem anno civitas 1111 Eodem anno ul. 1110 Civitas Laudensium Laudensis capta es!. tima ebdomada Ma.fuit capta in ultima eb. dii Laudenses capti fuerunt $\mathrm{d}$ oman a M adi i.

1120 fuit prima guerril 1120 Prima guerra Parme Prima guerra de Parma de Parma, qua Cremonenses et prelium ibi in glarea fuit et - preliun factum cum Parmensibus in Par-fuit de menae Junij, fuit in glarea prope a. nensi glarea conflixerunt.

Johanuem.

Da Sicard, wie gesagt, früher schriel, als die beilen Anualisteu, so kann über die Art des Quellenverhaltnisses kein Zweifel sein; er hat ebenfalls das verlorene Werkchen benutat. Ich bemerke noch, dass er das eine und andere Mal auch an Stellen, die in den Cremoneser Annalen fehlen, wit den Piacentiner übereinstimmt: 1084 ralida fames $=1085$ fumes valida; 1154 civitatem ecpit Asteusem, turres et muros destruens civitatis $=1154$ ad civitatem Asti perrexit et turres eiusdem ciritatis destruere fecit; 1153 Terdonam - cepit et funditus destruxit $=1154$ Terdonam - cepit et destruxit.

Das zweite Geschichtswerk, das heute auch untergegangen ist, verfasste ein Cremoneser im folgende» Jahrhundert. Seine Spuren können wir in anderen Werken verfolgeu, als in den bisher besprochenen. Iuhalt und Vergleichung werden unsere Kriterien sein.

Es muss auffallcn, wie oft in den wenigen Nachrichten, welche das Eigenthum der Chron. pont. et imp. Mantuana zu sein scheinen, rom Carrocio die Rede ist: man sollte meinen, der Verfasser babe sein ganzes kriegerisches Interesse auf dieses Fuhrwerk gerichtet Zum Jahre 1213 erzählt er, Cremona hätte das Carrocio ron Mailand 
erbeutet, und dasselbe sei uun ein Schnuck des Gemeindehauses von Cremona; zum Jahre 1237 höreu wir, wie das Carrocio von Mailand dem Kaiser in die Hände fiel, wie dieser es mit höhnenden Versen dem Papste sandte, wie die Römer es später in Stücke zerschlugen; 1248 ist es Cremona, das sein Carrocio an Parma verliert, aber 1249 gewinnt Cremona dafür das Carrocio von Parma, genannt Blanzardus. Genug, auf Einer Folioseite!) findet wan sechsmal das Wort "Carocium" und zwar in Berichten, die auf keine uns erhaltene Quelle zurückgehen. Meist handelt es sich dahei aber um Cremona: 1213 gewinut Cremona das Mailänder Carrocio, 1249 das Parmesaner, 1248 rerliert es das eigene; nur 1237 ist mit dem Carrocio, welches der Kaiser erbentet, der Name Cremona nicht unmittelbar verbunden; jedoch wissen wir ja, dass damals - es war in der Schlacht ron Cortenuova - Cremona auf Friedrichs Seite stritt und siegte.

Fast dieselbe Beobachtung machen wir nun aber auch in dem Werke des l'olosauus von Faenza und seines unbekannten Fortsetzers ${ }^{2}$. Nur besteht hier der Unterschied, dass allerdings das eine und audere Mal eiu (arrocio vorgeführt wird, ohne dass dabei von Cremona die Rede wïre. Dafür ist die Faentiner Chronik aber auch eine Arbeit vou viel grösserem Unfauge; im Vergleiche zum Volumen wird man die Erwähnungen eiues Carrocios, das nicht in einer Beziehung zu Cremona stände, nur als spärlich bezeichnen können. Umsomehr tritt das Carrocio hervor, wenn es sich um Cremona handelt. In c. 140 wird erzählt, wie 1213 das Carrocio der Mailänder von den Cremonesern erobert wurde und nun deren Gemeindehaus ziert; nach c. $1+1$ bekriegt die Feindin Cremonas, eben Mailaud, noch im selben Jahre dessell lreundin Pavia, aber es ergeht den Mailändern nur noch schlechter, „excepto quod nou amiserunt carrocium"; in c. 150 ist die Rede von der Schlacht bei Zibello, die 1218 geschlagen wurde: „et ibi fuerunt quatuor carrocia et obtinuerunt Cremouenses"; wie es c. 198 heisst, brechen 123.t zahlreiche Feiudo ins Gebiet von Cremoua ein, unter Anderem sind da auch "carrocia Mediolanensia et Brixiensia", aber auch C'remona hat seine Bundesgenossen, und es siegt "cum tribus carrociis". Wio man sieht, ist überall rom Carrocio die Rede, und Cremona steht im Mittelpunkt. Dagegen würde man sich vergebens bemühen, eine gleiche Hervorhebung des Carrocios der Faentiner oder der für Faenza so wichtigen Bologneser nachzuweisen: ich entsinue mich nur je einer Erwähnung ${ }^{3}$ ),

1) M. G. SS. XXIV. 218 Z. 48 bis 219 Z. 42.

589 flgg. 3) Cap. 158 p. 701. cap. 181 p. 725. 188 p. $i \geq 2$ des Carrocios vin Parma gedacht. $\left.{ }^{2}\right)$ Doc. di stor. Ital. VI. Ausserdem wird noch cap 
und beide geschahen nebenbei, ohne besondere Absicht. Nur ein einziges Mal noch wird der Carrocien - - wenn ich so sagen darf mit schärferer Accentuirung gedacht, während doch an der betreffenden Schlacht Cremona keinen Antheil hatte. Indess werden wir später sehen, wie trotzdem der Bericht gerade vom Standpunkte eines Cremonesers aus geschrieben sein kann.

Das gleiche Interesse führt nuu sofort zu der Vermuthung, dass in der Mantuaner und der Faentiner Chronik dieselbe Quelle benutat sei, und zwar eine Quelle Cremoneser Ursprungs. Man erwartet zur Vervollstïndigung des Beweises nur noch eime wörtliche Uebereinstimunung, wenu möglich gerade mit Rücksicht auf das Carrocio und Cremona. Daran fehlt es aber in keiner. Weise. Es ist das Verdienst von Waitz, welches in diesem Zusammenhange vatürlich sehr viel gilt, auf die Congruenzen hiugewiesen zu haben 1). Darunter ist von besonderen Werthe, dass der Mantuauer berichtet: „Cremonenses superaverunt Mediolanenses, eorum carocium, arma et scuta accipientes, quihus hodie palatium Cremonense decoratur", dass Tolosanus einen längeren Bericht über eineu Mailänder-Cremoneser Krieg mit den Worten beschliesst: „Mediolauelises carrocium amiserunt et armorum et scutorum multitudinem copiosam, uude hodie palatium Cremonensium decoratur".

Es versteht sich nun von selbst, dass unsere Autoren nicht blos Angabeu, die Cremona und zugleich das Carrocio betrafen, ihrer gomeinsamen Vorlage entlehnten. Mir kommt es besonders auf folgende Uebereinstinmung an:

Chron. pont. et imp. Mant. 218.

Cremonenses cum aliie Lumlardis de consensu douini Alexandri pape Mediolanum, revocatis undique habitatoribus, rehedificaverunt, et reducti sunt in civitatem. Eodem ivillesimo Cremona cum Me. diolano et Placentia contra civitatem Papiensen in eius confinio ex ho miuibus Guillelmi Montisferrati civitatem construxerunt, que ut fieret famosior, ab Alexandro papa III. Alexandrian vocaverunt.

Tolosan c. 60, 61.

Lombardi mala recolentes praeterita, vitare volentes futura deteriora, de consensudomini Alexandri papae Mediolanum, revocatis undi.

1) M. G. SS. XXIV. 214:, Aliquoties cum Tolosani Farentini chronico convenit, sed ex alio libro, fortasse Cremonensi, haec eum sumsisse putarim." Nebnnbei bemerkt, berührt die Uebereinstimmung, auf welche Waitz 219 Anm. s. aufmerksam macht, nicht mehr den Tolosan, sondern dessen Fortsetzer, welchen Tabarrini - Doc. di stor. ital. 698 Anm. - schon mit cap. 153 eintreten lässt. Uebrigens hätte Waitz die Congruenz auch schon zu Seite 215 Z. 52 und 58 hervorbeben können, denn da findet sich nochmals die gleichlautende Nachricht. 
que habitatoribus, reaedificarerunt. Anno domini 1167 civitate m in episcopatu Papiensi ex hominibus Guillelmi marchionis Montisfer. raticivitatem construxerunt, abdlexandroipsam Alexandriam nominantes.

Wie man sieht, hat Tolosan die Lokalfärbung völlig verwischt: weil sein Bericht sowohl die Wiederherstellung Mailands, wie auch die Gründung Alexandrias als ein Werk kurzweg der Lombarden bezeichnet, so könnte derselbe aller Orten geschrieben sein. Die beim Mantuaner erhaltene Fassung trägt dagegen ihren Cremoneser Ursprung gewissermassen an der Stiru: "Cremonenses cum aliis Lombardis etc.", "Cremona cum Mediolano et Placentia etc." Aber aus obiger Vergleichung ergibt sich wicht bloss, dass Tolosan das Allgemeiue an Stelle des Einzeluen setzt; mir ist hier wichtiger, dass der Cremoneser sich auch mit den Kämpfen Friedrichs I. gegen die Lombarden beschäftigte. Danach hat or unzweifelhaft auch dem Ausgange derselben seine Aufmerksamkeit zugewandt; und bier verweise ich nun auf die einzige oben noch übrig gebliebene Stelle, in der eines Corrocios - wie ich sagte - mit schärferer Accentuirung gedacht wird: Tolosan c. 80 widmet der Entscheidung bei Legnano nur sieben Zeilen und zweimal begegnet darin ein Carrocio.

Was den Bestand der neuen Quelle angeht, so hoffe ich, dass das Carrocio uns ein guter Wegweiser geworden ist; andererseits kanu natürlich die blosse Hervorhebung Cremonas, sofern sich dieselbe vom Staudpunkte eines Mantuaners und Faentiners nicht ron selbst versteht, einem etwaigen Versuche der Wiederherstellung nützliche Dienste leisten. Im Uebrigen entscheidet die Congruenz.

In Hinsicht der beiden zuletzt erwähnten Punkte möchte ich noch auf ein anderes Werk verweisen, als die bisher herang;ezogenen. Eben zu Faenza, wo also Tolosan und sein Fortsetzer schrieben, hat auch der Bologneser Cantinelli ein Geschichtsbuch verfasst, und wenn wun eive Abschrift der Cremoneser Chrouik, wie wegen der Benützung in der Faentiner wohl auzunehmen ist, sich zu Faenza vorfand, - was Wunder dann, wenn auch Cautinelli sich derselben bediente? Da fällt aber gleich eine Notic aus dem ersten der von ihm beschriebenen Jahre in dic Augen: „1228 in dicto praelio inter alios fuit mortuus dominus Puniamatus de Cremona, de nobilioribus et melioribus hominibus de Cremona"1). Cantinelli schrieh erst zu Ende des Jahrhunderts, und so möchte er kaum aus der mündlichen Tradition geschöpft haben. Die sich hieran knüpfende Vermuthung zu bestärken, bietet sich eine Congruenz mit

1) ap. Mittarelli Accessiones Faent. 2.1. 
der Chron. pont. et imp. Mant. dar, d. h. mit dem Werke, welches dem Cremoneser offenbar einen Theil soines Materials verdankt; freilich handelt es sich dabei nicht um Nachrichten von ausgesprochenem Cremoneser Uharakter, doch hat sich die veriorene Quelle ja auch nicht auf Cremona besehränkt. Zum Jahre 1235 benierkt Cantinelli: „Hoc anno frater Johannes de Vicencia de ordine praedicatorum incepit facere praedicationes, et fuit magna devocio"1), und beim Mantuaner Autor lesen wir: ,Eodem mense (sc. Madio 1235) frater Johanues de Vicentia ordinis predicatorum cepit facere sollempnes predicationes etpaces, et fuit magna derotio"s).

Wenn diese Combination richtig ist, so würde die wörtliche Uebereinstinumung beweisen, dass der Cremoneser sein Werk, welches also auch Cantinellis Quelle gewresen wäre, jedenfalls erst nach 1235 beendet hat. Schon weiter aber, als die Congruenz führte uns die blosse Erwähnung Cremonas und des Carrocios in der Chronik des Mantuauers, nämlich bis 1248. Und von dieser Seite ergibt sich nun eine Schwierigkeit, die gehoben werden muss, oder das gewonnene Resultat bedarf der Correctur. Wie kaun nämlich eine Quelle bis 1248 und z.war zum Mindesten bis dahin gereicht haben, derweil doch Tolosan aus ihr geschöpft haben soll, Tolosan, d. h. ein Autor, der 1226 eivem langen Siechthum erlag ${ }^{3}$ ), den schon 1219 ein Schlag Verstand und Sprache geschwächt hatte?') Ferner, das Werk seines Fortsetzers reicht nur bis 1237, und auch er soll sich der viel spïter endenden Chrouik des Cremonesers bedient haben.

Da bemerke ich nun, dass trotz der Verschiedenheiten, die der jüngste Herausgeber vor und uach 1217 beobachtet hat ${ }^{5}$ ), sich doch auf der anderen Seite die einheitliche Redaktion nicht verkenuen lässt. Ess kann ja nicht meine Aufgabe sein, über die Composition der Faentiner Chronik zu handeln, die auffallenden Gleichbeiten der ersten und zweiten Hälfte vor Augen zu führen; ich beschräuke mich darauf, zwei Stilproben hervorzuheben, und zwar vergleiche ich Sätze des früheren Theiles, deren Kern offeubar Cremoneser Ursprungs ist, - eben diese vergleiche ich mit Sätzen des späteren Theiles $\left.{ }^{6}\right)$. Weun

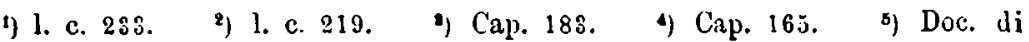

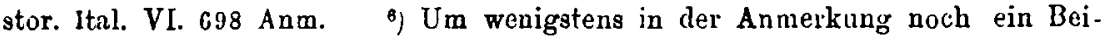
spiel anzufübren, so vergleiche ich cap. 141 p. $69 \hat{s}$ ao. 1213 mit cap. 170 p. 712 ao. 122s. ,Et ibi deterius habuerunt, quam hostibus intulissent, excepto quod non amiserunt carrocium." - , igne apposito in domibus burgi, $u b i$ de. terius habuerunt, quam hostibus intulissent." 
sich dabei eine charakteristische Gleichheit der Schreibart ergiebt, wenn also eine Umarbeitung stattgefunden hat, so können die Cremoneser Bestandtheile selbst recht wohl damals erst hinzugekommen sein, sei es durch den Fortsetzer, der ja nicht gerade im Jahre 1237, dem Endpunkte seines Werkes, die Ereignisse eben dieses Jahres auch dargestellt haben muss, [der vielmehr geraume Zeit später Notizen und Erinnerungen verarbeitet haben kann, sei es in noch fernerer Zeit durch einen Anderen, der dann zugleich auch der zweiten Hälfte eine neue Redaktion gegeben hätte.

Chron. pont. et imp. Mant. 218.

Cremonenses cum aliis Lumbardis de consensu domini Alerandri pape Mediolanum, revocatis undique habitatoribus, rehedifi. caverunt, et reducti sunt in civitatem. Eodem millesimo Cremona cum Mediolano et Placentia contra civitatem Papiensem in eius confinio e $\mathbf{x}$ hominibus Guillelmi Montigferrati civitatem construxerunt, que ut fieret famosior, a b Alesandro papa III. Alesandriam vocaverunt.

Tolsan. a 60.61. p. 658 .

Lombardi mala recolentes praterita, vitare volentes futura deteriora, d e co nsensu domini Alexandri papae Mediolanum, revocatio undique babitatoribus reaedificaverunt. Anno domini 1167 civitatem in episcopatu Papiensi ex hominibus Guillelmi marchionis Montisferrati construxerunt, ab Alexandro ipsam Alexandriam nominantes cum iuraverunt praeterea fere onnes Lombardi, - contra imperatorem se invicem iuvaturos, si imperator vel eius nuncius aliquid vellet pertractare iniuste.

Contin. c. 181. c. 180. p. 719. 717.

Illico rectores Lombardiae, mala recolentes praeterita, volentes futura oitare deteriora etc. - omnes fere Lombardi contra imperatorem coniuravere se invicem iuvaturos, dummodo imperator vel eius nuncius aliquem vellet pestractare iniuste.

Dass derselbe Autor, dessen Geist aus den Wendungen der Fortsetzung spricht, auch in dem Theile, welcher durchweg als das Eigenthum des Tolosan gilt, die Feder geführt hat, scheint mir die Vergleichung zu beweisen. Und dieser nuu, sei er der Fortsetzer, der dann aber verhältnissmässig spät geschrieben haben müsste, sei es ein beliebiger Redaktor, kann doch auch die Cremoneser Bestandtheile selbst hinzugefügt haben.

So hätten wir denn in Hinsicht der Cremoneser Geschichtschreibung nicht blos den Verlust jener Chronik des Priesters Johannes zu beklagen, auch noch andere historische Aufzeichnungen hat die Cngunst der Zeit getroffen. Es ist ein geringer Trost, deren Spuren in späteren W trken oder Bearbeitungen nachgewiesen zu haben, aber immerhin gewinnen die erhaltenen Reste doch an Werth, wenn man ihre Natur erkannt hat. 